\title{
Reflexão sobre políticas públicas e estratégias na saúde integral da criança
}

\author{
Mirna Albuquerque Frota ${ }^{1}$ \\ Julyana Almeida Maia² \\ Aline de Souza Pereira ${ }^{3}$ \\ Caroline Soares Nobre ${ }^{4}$ \\ Luiza Jane Eyre de Souza Vieira 5
}

\begin{abstract}
Este artigo representa uma reflexão crítica sobre os programas e políticas públicas voltados para as crianças, numa retrospectiva da Constituição de 1988 até os documentos atuais, confrontando autores e documentos oficiais do Ministério da Saúde com o objetivo de abordar e descrever a situação atual de desenvolvimento das políticas públicas brasileiras de saúde infantil e juvenil. Conclui-se que a criança deve ser considerada de maneira holística, envolvendo aspectos internos e externos como o ambiente em que vive, o que requer uma visão interdisciplinar e o exercício da intersetorialidade, fatores fundamentais para garantir a integralidade e efetividade das ações.
\end{abstract}

Descritores: Políticas Públicas, Direitos da Criança, Saúde da Criança.

\section{Reflection on public policies and strategies for child's integral health}

This article represents a critical reflection on public policies and programs aimed at children, in a retrospective of the Constitution of 1988 to current documents, comparing the authors and official documents of the Ministry of Health with the objective of addressing and describe the current state of development of Brazilian public policies for child and adolescent health. We conclude that the child should be considered in a holistic manner, involving internal and external aspects such as the environment in which it lives, requires an interdisciplinary approach and the exercise of intersectionality, which is crucial to ensure the completeness and effectiveness of actions.

Descriptors: Public Policy, Children's Rights, Child Health.

\section{Reflexión sobre políticas públicas y estrategias para la salud integral del niño}

Este artículo constituye una reflexión crítica sobre las políticas públicas y programas dirigidos a los niños, una retrospectiva de la Constitución de 1988 para presentar los documentos, comparación de los autores y los documentos oficiales del Ministerio de Salud con el objetivo de abordar la situación actual y describir el desarrollo de las políticas públicas brasileñas para la salud del niño y del adolescente. Concluimos que el niño debe ser considerado en forma holística, involucrando aspectos internos y externos, tales como el medio ambiente en que viven, requiere un enfoque interdisciplinario y el ejercicio de la interseccionalidad, que es crucial para garantizar la integridad y eficacia de las acciones.

Descriptores: Política Pública, Derechos de los Niños, Salud de los Niños.

\section{INTRODUÇÃO}

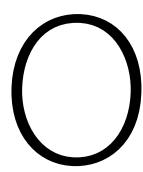

fracasso e, sobretudo, a perda de legitimidade do modelo de atendimento à crianças e adolescentes em situação de risco, vigente até meados da década de 1980, baseado na doutrina da situação irregular e expresso no Código de Menores, colocou no campo dessas políticas novas possibilidades e problemas. Desse modo, para que as ações de promoção da saúde sejam eficazes, deve-se tratar de formar igualitária todos os que buscam a saúde ${ }^{(1-2)}$.

Ressalta-se que o século 20 marcou avanços significativos sobre a visão da criança como cidadão com capacidade de exercer seus direitos e deveres. A infância passa a ser reconhecida como etapa extraordinária para a formação de uma vida adulta futura ${ }^{(3)}$. Tais mudanças somam formulações, projetos, programas e políticas, com esforço do governo e sociedade, de transpor letras e documentos em ações integradas que favoreçam a criança e o adolescente.

O Brasil representa um dos 160 países participantes da Declaração Mundial sobre a Sobrevivência, a Proteção e o Desenvolvimento da Criança, pactuada em 1990(4). É relevante registrar que os movimentos estratégicos, a experiência acumulada e as pesquisas realizadas estiveram até bem pouco tempo atrás focadas no objetivo de alterar um quadro

1 Enfermeira. Doutora em Enfermagem pela Universidade Federal do Ceará - UFC. Docente do Mestrado em Saúde Coletiva e do Curso de Graduação em Enfermagem da Universidade de Fortaleza - UNIFOR. Coordenadora do Grupo de Pesquisa: Núcleo de Pesquisa e Estudo em Saúde da Criança - NUPESC - E-mail: mirna@unifor.br 2 Fisioterapeuta. Mestranda em Saúde Coletiva e Docente do Curso de Graduação em Fisioterapia da Universidade de Fortaleza - UNIFOR.

3 Enfermeira. Mestranda em Saúde Coletiva pela Universidade de Fortaleza - UNIFOR. Bolsista do CNPq. Membro do Núcleo de Estudo em Acidentes e Violência - NEPAV. 4 Aluna do $8^{\circ}$ Semestre do Curso de Graduação em de Enfermagem da Universidade de Fortaleza - UNIFOR. Bolsista PIBIC/CNPq. Membro do Núcledo de Pesquisa e Estudo em Saúde da Criança-NUPESC/UNIFOR/CNPq.

5 Enfemeira. Doutora em Enfermagem pela Universidade Federal do Ceará - UFC. Docente do Mestrado em Saúde Coletiva e do Curso de Graduação em Enfermagem da Universidade de Fortaleza - UNIFOR. Coordenadora do Núcleo de estudo em Acidentes e Violência - NEPAV. 
sanitário marcado por elevados índices de desnutrição e morbimortalidade por causas infecciosas. Esse objetivo moldou a formação e o perfil das equipes técnicas nas diferentes instâncias de gestão do sistema de saúde e a produção de conhecimento a esses grupos etários ${ }^{(5)}$.

Muitas ações são desempenhadas a fim de mudar a situação da criança no Brasil e no mundo mas, mesmo com tantas iniciativas, não se conseguiu obter índices realmente satisfatórios, portanto a relevância do incentivo "precoce" à criança. Precoce no sentido de atuar desde cedo e de manter o incentivo por toda a infância e a vida. É evidenciado que o sujeito, quando adulto, é consequência do que foi quando criança.

Reconhecendo a importância e a incipiência das políticas públicas para a saúde integral da criança, este texto revisita documentos oficiais com o objetivo de abordar e descrever o desenvolvimento de Políticas Públicas de Saúde Infantil no Brasil. Desse modo, discorre sobre o Estatuto da Criança e do Adolescente (ECA), Agenda de Compromissos para a Saúde Integral da Criança e Redução da Mortalidade Infantil, Plano Nacional para Primeira Infância (PNPI) e Política de Saúde Mental Infantil.

\section{O ESTATUTO DA CRIANÇA E DO ADOLESCENTE (ECA)}

O ECA apresenta-se como evolução da Constituição de 1988 e do código do menor infrator. Criado em 1990 como lei ${ }^{\circ}$ 8069 e atualizado em 2003, passa a tratar a criança e o adolescente sob a ótica pessoal e social, outorgando-lhes vez e voz diante das decisões políticas vigentes da época e
“Em 2010, o Ministério da Saúde publicou que o Brasil mantém queda sustentada de mortalidade infantil. Entre 1990 e 2008, a mortalidade neonatal caiu 36\%" gestante, através do Sistema Único de Saúde, o atendimento pré e perinatal; Art. 9. O poder público, as instituições e os empregadores propiciarão condições adequadas ao aleitamento materno, inclusive aos filhos de mães submetidas a medida privativa de liberdade; Art. 10. Os hospitais e demais estabelecimentos de atenção à saúde de gestantes, públicos e particulares, são obrigados a: [...] V manter alojamento conjunto, possibilitando ao neonato a permanência junto à mãe $e^{(6)}$.

A taxa de mortalidade entre menores de 1 ano no Brasil apresenta queda contínua desde 1990, com redução de $59,7 \%$ no período de 2007. Esse declínio é resultado do aumento da cobertura vacinal, terapia de reidratação oral, ampliação dos serviços de saúde como pré-natal, redução da fecundidade, melhoria das condições ambientas e aumento do grau de escolaridade das mães e das taxas de aleitamento materno. Registra-se, igualmente, o decréscimo acentuado na mortalidade pós-neonatal, decorrente de causas como gastroenterites, infecções respiratórias e má nutrição protéico-calórica. Esse decréscimo foi acompanhado pelo aumento da participação relativa da mortalidade neonatal na composição do indicador, quando predominam as afecções originadas no período perinatal(9-10).

Em 2010, o Mistério da Saúde publicou que o Brasil mantém queda sustentada de mortalidade infantil. Entre 1990 e 2008 , a mortalidade neonatal caiu $36 \%$ (a quantidade de óbitos baixou de 46.893 para 29.881). No mesmo período, a mortalidade infantil geral (de 0 até 12 meses de vida) estabelecendo que transgressões cometidas sejam tratadas com atitudes socioeducativas ${ }^{(6)}$. O fundamento da lei está no artigo 227 da Constituição de 1988:

"É dever da família, da sociedade e do Estado assegurar à criança e ao adolescente, com absoluta prioridade, o direito à vida, à saúde, à alimentação, à educação, ao lazer, à profissionalização, à cultura, à dignidade, ao respeito, à liberdade e à convivência familiar e comunitária, além de colocá-los a salvo de toda forma de negligência, discriminação, exploração, violência, crueldade e opressão".

A criança é contemplada sob uma visão holística, como ser humano em desenvolvimento, abrangendo diversas áreas da vida infância. Os direitos assegurados poderiam estar subentendidos em um único: à saúde. Isso pode ser justificado quando se considera saúde como o complemento para o bemestar físico, mental e social proposto pela Organização Mundial de Saúde (OMS).

O estatuto declara como fundamentais os direitos à vida e à saúde. E considera a criança como pessoa humana ainda dentro do ventre materno. Dessa perspectiva, foram contempladas conquistas do Programa Nacional de Saúde Materno-Infantil e do Programa de Prevenção à Gravidez de Alto Risco(7-8).

Segundo a lei em questão: Art. 8. É assegurado à teve redução de 54\%: o número de mortes caiu de 95.476 para 43.601. Por fim, observou-se mudança na caracterização da mortalidade infantil no país ${ }^{(11)}$. Os dados sinalizam o impacto das ações de promoção à saúde direcionada a mãe-filho, porém, a população infantil ainda não usufrui de direitos conquistados e promulgados em documentos oficiais e pactuações realizadas, entre nações, para a melhoria da saúde infantil.

\section{AGENDA DE COMPROMISSOS PARA A SAÚDE INTEGRAL DA CRIANÇA E REDUÇÃO DA MORTALIDADE INFANTIL}

É assegurado atendimento médico à criança e ao adolescente, por meio do Sistema Único de Saúde (SUS), garantido o acesso universal e igualitário às ações e aos serviços para promoção, proteção e recuperação da saúde(6).

Foi criada a Agenda de Compromissos para a Saúde Integral da Criança e Redução da Mortalidade Infantil como estratégia para fazer valer esse direito, que consiste na recomendação para as ações voltadas para criança, ressaltando a importância do cuidado integral e multiprofissional.

Ressalta-se que a Agenda tem por finalidade apoiar a organização da assistência à população infantil e possibilitar que os gestores e profissionais de saúde identifiquem as ações prioritárias para a saúde da criança. Propõe a definição 
de diretrizes para identificação de linhas de cuidado na qual são denominadas como: nascimento saudável, crescimento e desenvolvimento, distúrbios nutricionais e doenças prevalentes na infância, que devem constar no cardápio básico para o funcionamento adequado dos serviços e de toda a rede de ações de saúde da criança no nível local, de maneira a prover respostas mais satisfatórias para essa população(12-13).

Para seguimento da criança, são utilizados manuais do Ministério da Saúde pertencentes a programas preexistentes, como o Protocolo de Pré-Natal de Baixo Risco, Acompanhamento do Crescimento e Desenvolvimento Infantil e ação as Doenças Prevalentes da Infância.

\section{PLANO NACIONAL PARA PRIMEIRA INFÂNCIA (PNPI)}

Nesse cenário de preocupação encontram-se o Estado, as Organizações não-governamentais (ONGs) e a sociedade no tocante da qualidade de vida na infância, culminando em 2008 no esboço do PNPI, determinando diretrizes e metas que devem ser tomadas pelo Estado para fazer valer os direitos da criança na faixa etária até os 6 anos de idade.

O Plano Nacional pela Primeira Infância constitui, sobretudo, uma expressão da vontade nacional de cumprir os compromissos internacionais assumidos pelo país em documentos como a Convenção dos Direitos da Criança, das Nações Unidas, o Plano de Educação de Dacar 2000/2015 e os Objetivos do Milênio, de que o Brasil é signatário e com os quais está comprometido. Articula, sobretudo, com outros planos e compromissos nacionais, como o Plano Nacional de Educação (2001/2011), Plano Nacional de Saúde, Plano Nacional de Assistência Social, Plano Nacional de Cultura, Plano Nacional de Combate à Violência contra a Criança, Plano Nacional de Promoção, Proteção e Defesa do Direito de Crianças e Adolescentes à Convivência Familiar e Comunitária etc. e se atualizará e complementará com outros planos que venham a ser elaborados ${ }^{(14)}$.

É válido ressaltar que o plano enfatiza a comprovação científica da necessidade de garantir desenvolvimento saudável nos primeiros anos de vida como investimento para a formação de adultos preparados a melhorar a capacidade intelectual e tornar a sociedade brasileira próspera e produtiva.

A miséria alimenta um ciclo vicioso a cada geração, interligando baixo nível de escolaridade, desemprego, criminalidade, gravidez na adolescência, desnutrição e altos índices de mortalidade infantil. Essa realidade é percebida quando mães de desnutridos relatam utilizar o leite recebido no posto de saúde como moeda de troca para vencer as consequências do desemprego e apresentam como barreira para não trabalharem fora de casa fatores como: não ter com quem deixar os filhos e uma nova gravidez ${ }^{(15)}$.

O Brasil possui 31,2\% de famílias com menores de até 6 anos que vivem com renda per capita de até meio salário mínimo. Esse dado expressa a vulnerabilidade social da população infantil(3).

Ressalta-se que o Programa Bolsa Alimentação, criado em 2004, existe com a finalidade de minimizar os efeitos da pobreza, fornecendo apoio financeiro para gestantes, nutrizes e crianças de 6 meses a 6 anos, cuja renda familiar seja inferior a meio salário mínimo ${ }^{(16)}$. A faixa etária escolhida respeita o período de amamentação exclusiva, no qual mulher e bebê estão protegidos pela Política Nacional de Incentivo ao Aleitamento Materno, formulada em 1981 e reafirmada pela Constituição de 1988 e pelo ECA.

Porém, como observado com o programa do leite destinado à criança, constata-se com frequência que esse benefício é utilizado para contemplar as necessidades de outros membros da família. Esse fator agrava-se pelo baixo nível de instrução materna, relacionado com maus hábitos alimentares e de cuidados em geral ${ }^{(15)}$.

\section{POLÍTICA DE SAÚDE MENTAL INFANTIL}

Em 2001, antes da existência de políticas específicas para saúde mental infantil, foram criados os Centros de Atenção Psicossocial Infanto-Juvenis (Capis), com serviços de natureza pública, formados por equipe interdisciplinar.

O Capis é composto de equipes multiprofissionais, contendo, no mínimo, um psiquiatra, um neurologista ou pediatra com formação em saúde mental infantil, um enfermeiro, quatro profissionais de nível superior, entre psicólogo, assistente social, terapeuta ocupacional, fonoaudiólogo, pedagogo, além de cinco profissionais de nível médio. A inclusão tardia da saúde mental na infância e adolescência decorre da dificuldade do diagnóstico e do tempo de início da manifestação dos sinais e sintomas, sobretudo o crescimento insuficiente dos Capis em relação à demanda ${ }^{(17)}$.

O Fórum Nacional de Saúde Mental Infantil, ocorrido em 2004, assegurou a importância do desenvolvimento de ações e discussões que favoreçam a reforma psiquiátrica para essa população específica ${ }^{(18)}$. Foram estudadas as necessidades de consolidação da Política de Saúde Mental Infantil Juvenil e o reconhecimento pelo Sistema Único de Saúde dos serviços de prevenção, promoção e proteção objetivando a saúde mental.

É válido salientar que, com a divulgação de fatores de risco para deficiência mental ou indicadores de saúde, surgirão demandas de atendimento que devem ser acompanhadas por centros e profissionais especializados para absorver essa clientela emergente.

\section{CONSIDERAÇÕES FINAIS}

O texto apresentou os avanços registrados com elaboração e implantação de políticas, planos e estatutos que norteiam ações intersetoriais direcionadas à saúde integral da criança. Retratou conceitos, medidas de ação, projetos e reflexões críticas à situação vigente. 
O compromisso do Estado em incluir a criança nas decisões políticas, expresso nos acordos de contexto mundial e no incentivo à sociedade e aos setores público e privado para desenvolver ações que beneficiem mãe-filho, tem sido reconhecido e impactado nos indicadores de qualidade de vida da criança.

A preocupação com a redução da mortalidade infantil foi ampliada visando a proporcionar ao ser em desenvolvimento melhor qualidade de vida. Esse é um tema complexo que envolve a articulação de diferentes áreas ligadas ao indivíduo, família, sociedade e o poder público.

Do mesmo modo, estudos científicos, sobretudo sobre a saúde de crianças e o alcance de metas, operacionalização de programas e efetivação de políticas de saúde, mostram que a qualidade de vida e o alcance da saúde exigem mudanças nas intervenções e avaliações do modelo de atenção centrado na doença, para a abordagem individual, com respeito às singularidades da criança e, sobremodo, centrada na família. Contudo, essas ações intersetoriais e intrasetoriais ainda não repercutiram na tão desejada saúde integral, pois o tema, em sua complexidade, requer a articulação de diferentes áreas dos saberes e de decisões que assumam a saúde integral da criança como uma política de Estado e, não apenas, como plataformas de governos que se mostram insustentáveis e desarticuladas do bem coletivo.

Considerar a criança de maneira holística envolve concepções macro e micropolíticas dos planejadores e gestores do cuidado indireto e direto. Aos que abraçam e compactuam com essa ideia, esforcem-se para concretizar ações interdisciplinares, pois são fundamentais para garantir a integralidade e efetividade da atenção à saúde da criança.

Importante referir que o seguimento à saúde da criança se concretizará mediante efetiva prática social, conclamando-se a co-participação dos sujeitos e ampliando-se as dimensões do cuidado para que os profissionais percebam a criança como possuidora de direitos e de usufruir de um cuidado integral. O profissional enfermeiro é um dos que se encontra instrumentalizado para ofertar este cuidado integral, destarte sua história e formação profissional ter sido alicerçada em teorias humanísticas.

\section{Referências}

1. Costa BLD, Carneiro CBL. Os desafios da inclusão social: programas de assistência a infância e juventude vulnerabilizada na administração municipal de Belo Horizonte. Belo Horizonte: Fundação João Pinheiro, Escola de Governo; 2004. (Caderno de Textos).

2. Aquino OS, Ximenes LS, Pinheiro AKB. Políticas públicas voltadas á atenção à prostitutas: breve resgate histórico. Rev Enferm Foco. 2010;1(1):18-22.

3. Ministério da Saúde (BR). Secretaria de Políticas de Saúde. Área da Saúde da Criança. Programas e projetos da saúde da criança: responsabilidades compartilhadas em benefício das crianças brasileiras. Rev Bras Saúde Matern Infant. 2002;2(2):193-200.

4. Ministério da Saúde (BR). Secretaria de Políticas de Saúde. Área da Saúde da Criança. Programas e projetos da saúde da criança: responsabilidades compartilhadas em benefício das crianças brasileiras. Rev Bras Saúde Matern Infant. 2002a;2(3):319-22.

5. Gomes MASM. As políticas públicas na área da criança. Ciênc Saúde Coletiva. 2010:15(2).

6. Ministério da Saúde (BR). Estatuto da Criança e do Adolescente. 2a ed. Brasília: Ministério da Saúde; 2003.

7. Ministério da Saúde (BR). Secretaria Nacional de Saúde. Coordenação de

Proteção Materno-Infantil. Programa Nacional de Saúde Materno-infantil. Brasília: Ministério da Saúde; 1975.

8. Ministério da Saúde (BR). Programa de prevenção à gravidez de alto risco. Brasília: Ministério da Saúde; 1978.

9. Ministério da Saúde (BR). Portal Saúde. Diagnóstico do Brasil, mortalidade em declínio [Internet]. [citado em 2009 Set 2]. Disponível em: http://portal.saude.gov. br/portal/saude/profissional/visualizar_texto.cfm?idtxt=32203\&janela=1 10. Duarte CMR. Reflexos das políticas de saúde sobre as tendências da mortalidade infantil no Brasil: revisão da literatura sobre a última década. Cad
Saúde Pública. 2007;23(7):1511-28.

11. Ministério da Saúde (BR). Portal Saúde. Brasil mantém queda sustentada de mortalidade infantil [Internet]. [citado em 2010 Ago 1]. Disponível em: http://portal.saude.gov.br/portal/aplicacoes/noticias/default. cfm?pg=dspDetalheNoticia\&id_area=124\&CO_NOTICIA=11571

12. Ministério da Saúde (BR). Agenda de Compromissos Integral da Saúde da Criança e Redução da Mortalidade Infantil. Brasília: Ministério da Saúde; 2004

13. Villar MAM, Wuillaume SM, Cardoso MHCA. Perspectivas de médicos do Programa Saúde da Família acerca das linhas de cuidado propostas pela Agenda de Compromissos Integral da Saúde da Criança e Redução da Mortalidade Infantil. Cad Saúde Pública. 2009;25(2):349-58.

14. Executiva do Programa Comunidade Solidária (BR). Comitê da Primeira Infância. Comitê da Primeira Infância: esboço Plano Nacional pela Primeira Infância [Internet]. [citado em 2009 Set 22]. Disponível em: http://www. primeirainfancia.org.br

15. Frota MA, Barroso MGT. Repercussão da desnutrição infantil na família. Rev Latinoam Enferm. 2005;13(6):996-1000.

16. Ministério da Saúde (BR). Departamento de Apoio à Descentralização. Comissão Intergestores Tripartite. Resumo executivo. Reunião extraordinária em 21 de Agosto de 2001. Bolsa Alimentação [Internet]. [citado em 2009 Set 22]. Disponível em:

http://portal.saude.gov.br/portal/arquivos/pdf/resumo_jun_01.pdf 17. Couto MCV, Duarte CS, Delgado PGG. Saúde mental infantil na saúde pública brasileira: situação atual e desafios. Rev Bras Psiquiatr. 2008;30(4):384-89. 18. Ministério da Saúde (BR). Caminhos para uma política de saúde mental infanto-juvenil [Internet]. [citado em 2005 Ago 15]. Disponível em: http://dtr2001.saude.gov.br/editora/produtos/livros/pdf/05_0379_M.pdf 


\section{NO QUE DEPENDER DA GENTE, A DENGUE VAI VIRAR PEÇA DE MUSEU.}

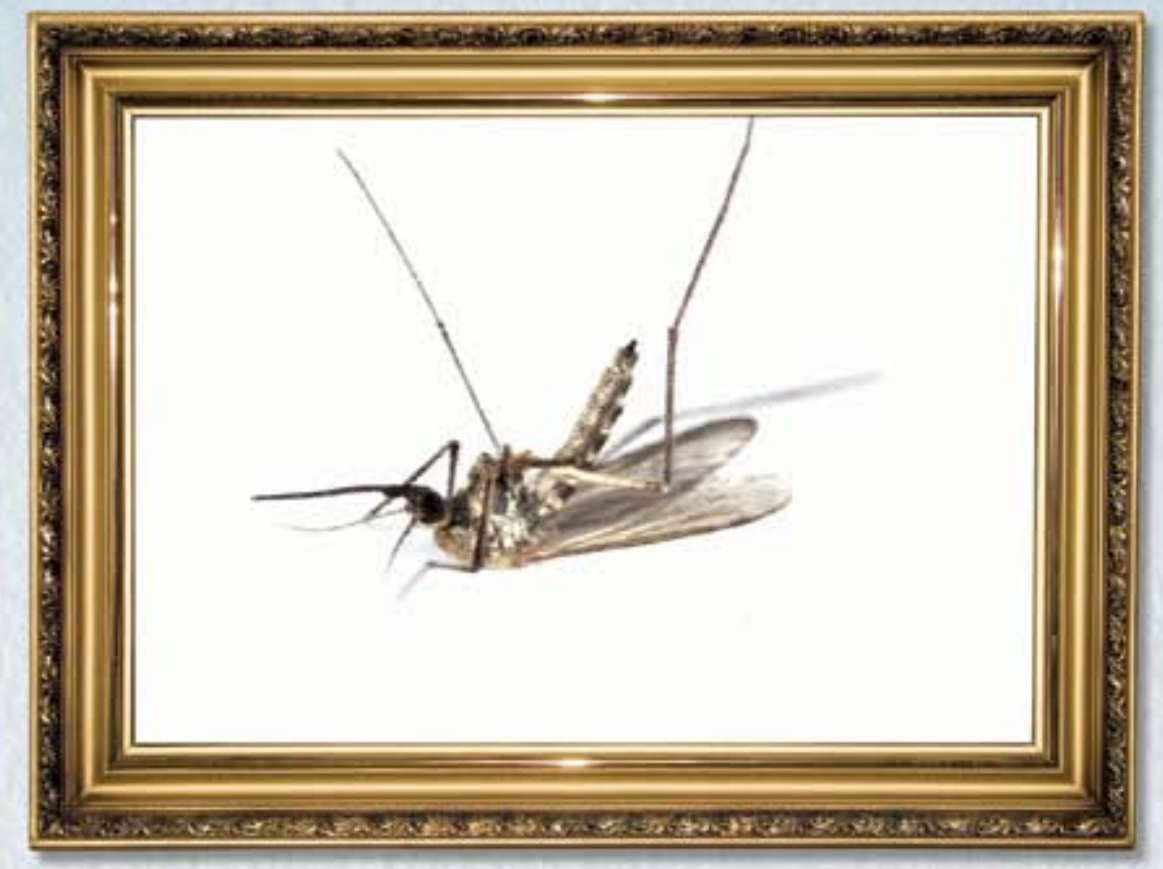

O Museu Nacional de Enfermagem Anna Nery apresenta ao público a exposição "DENGUE NUNCA +". Uma mostra de curta duração com o objetivo de alertar para os perigos da doença e fomentar no público de diversas idades hábitos cotidianos de combate à proliferação do mosquito.

A exposiçào "Dengue Nunca +" foi concebida pelo Museu Nacional de Enfermagem Anna Nery para contribuir na formaçầo de uma consciència coletiva de prevenção à doença, esclarecendo suas principais causas e consequềncias.

Ao mesmo tempo, demonstrando, por meio de açỏes simples e cotidianas, ser possivel transformar esta realidade e disseminar hábitos saudáveis e de prevenção em diversos ambientes de convivio.

Venha ver de perto a exposição e saber o muito que juntos podemos fazer.

Programação

Novembro a fevereiro - Terça a sexta, das $9 \mathrm{~h} 30$ às $17 \mathrm{~h} 30 /$ Sábado, das $13 \mathrm{~h}$ às $17 \mathrm{~h} 30$
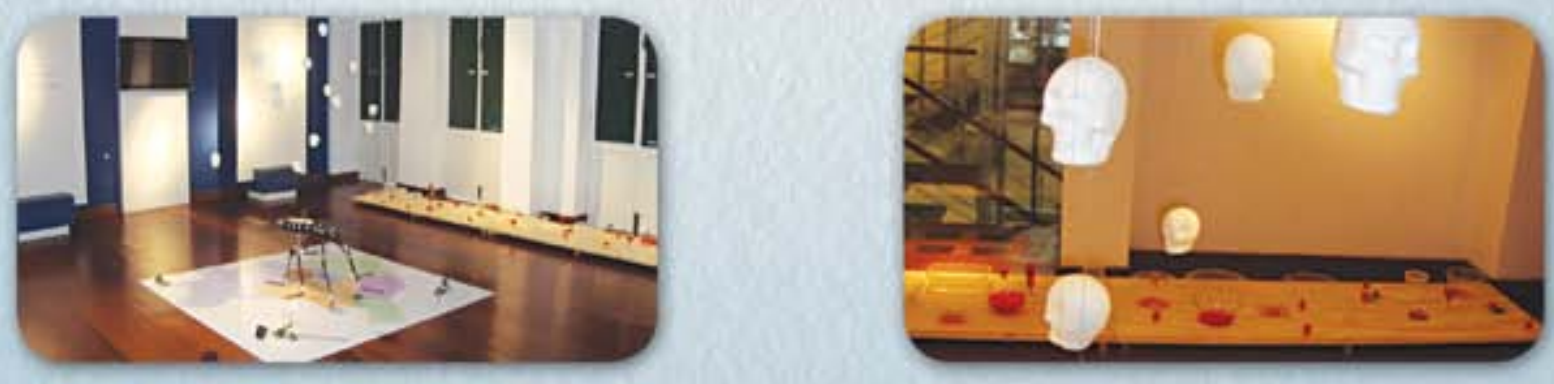

Iniciativa e patrocínio:

Realizaçio: 